\title{
Candida tropicalis in the Peripheral Blood of a Surgical Patient
}

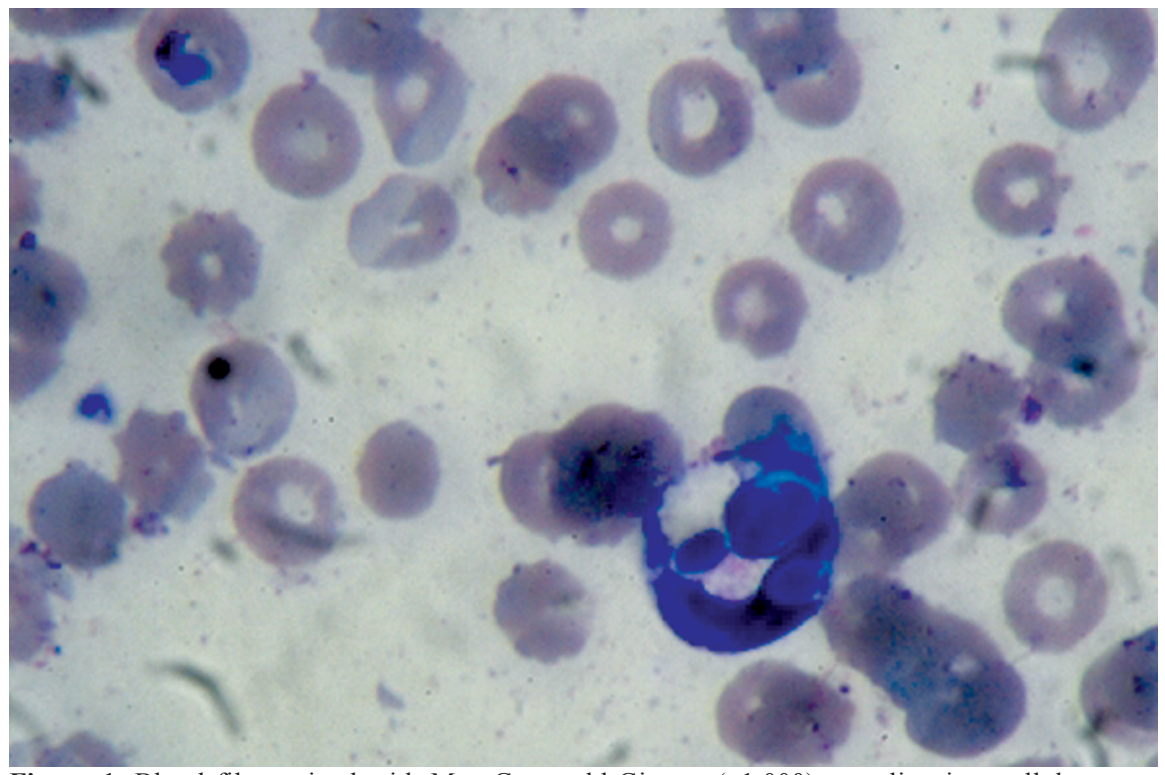

Figure 1. Blood film stained with May-Grünwald-Giemsa $(x 1,000)$ revealing intracellular yeastlike organisms. Klebsiella pneumoniae and Enterococcus faecalis. One of the samples was also positive for yeasts, which were later identified as Candida tropicalis. Her hemoglobin, leucocyte count, and platelet count were $6.7 \mathrm{~g} / \mathrm{dL}, 3,370 / \mathrm{mL}$ and 36,000/mL, respectively. Most of her leucocytes were polymorphonuclear cells (91\%). Yeasts were abundant in a blood film; they were being phagocytized (Figure 1).

Candida spp. are rarely seen in peripheral blood smears [1]. Only a handful of cases of disseminated candidosis diagnosed from peripheral blood smears were included in the study by Yera et al., published in 2004 [2]. Interestingly, most cases were caused by Candida albicans and involved patients with intestinal obstruction. It appears that this can only occur when fungal elements are present in large numbers in the peripheral blood [3]. For instance, it has been demonstrated that yeasts need to be at a concentration of at least $5 \times 10^{5} \mathrm{CFU} / \mathrm{mL}$ before they can be seen in the peripheral blood by experienced pathologists [4]. A 1,000-fold higher concentration would probably be required for non-experts during routine slide review. Since most cases of candidemia are associated with much lower concentrations of circulating yeasts (usually $<100 \mathrm{CFU} / \mathrm{mL}$ ) [4], early detection of candidemia is unlikely in the vast majority of patients, when using this method. However, in some cases, as we report here, a review of blood smears could lead to an early diagnosis, sometimes days before culture result. Unfortunately, our patient died a few hours after her blood was obtained, because of both severe underlying disease and acute illness.

\section{References}

1. Nadir E., Kaufshtein M. Images in clinical medicine. Candida albicans in a peripheral-blood smear. N Engl J Med 2005;353:e9.

2. Yera H., Poulain D., Lefebvre A., et al. Polymicrobial candidaemia revealed by peripheral blood smear and chromogenic medium. J Clin Pathol 2004;57:196-8.

3. Berrouane Y., Bisiau H., Le Baron F., et al. Candida albicans blastoconidia in peripheral blood smears from non-neutropenic surgical patients. J Clin Pathol 1998;51:537-8.

4. Branda J.A., Ferraro M.J., Kratz A. Sensitivity of peripheral blood smear review for the diagnosis of Candida fungemia. Arch Pathol Lab Med 2007;131:97-101.

The Brazilian Journal of Infectious Diseases (www.bjid.com.br)

2009;13(1):80.

(C) 2009 by The Brazilian Journal of Infectious Diseases and Contexto Publishing. All rights reserved. 